\title{
Morphological and attachment changes of Lamellodiscus theroni (Monogenea: Diplectanidae) during its post-larval development on fish
}

\author{
Neus Sánchez-García, Juan A. Raga and Francisco E. Montero
}

Cavanilles Institute of Biodiversity and Evolutionary Biology, Science Park, University of Valencia, Paterna, Spain

\begin{abstract}
Species of the genus Lamellodiscus Johnston et Tiegs, 1922 (Monogenea: Diplectanidae) are characterised by a complex haptor bearing many different attachment elements: two pairs of main hooks joined by medial bars, 14 peripheral marginal hooks and one or two lamellodiscs, formed by several overlapping sclerotised plates (lamellae). These haptoral structures appear gradually during parasite development and, therefore, attachment strategies vary with developmental stage. The main aim of this work was to study the developmental changes of Lamellodiscus theroni Amine, Euzet et Kechemir-Issad, 2007 under experimental conditions, with special attention to the gradual variations in attachment strategies and the pathological implications. Throughout the gradual development of the sclerotised structures, six developmental phases were distinguished in L. theroni: phase I, with only 14 peripheral marginal hooks; phase II, with main hooks (ventral and dorsal) formed; phase III, with ventral bar formed; phase IV, with dorsal bars formed; phase V, with dorsal and ventral lamellodiscs formed; and phase VI, adult stage with male copulatory organ formed. During development, parasites attach to different parts of the first and secondary gill lamellae and the mode of attachment changes from unspecific stage, i.e. based on piercing any flat gill tissue in the early stages, through an intermediate stage when ventral and dorsal main hooks are completely functional and parasites become restricted to the interlamellar space, and finally to the definitive adult attachment stage when lamellodiscs are fully developed. The timing of key events in the development of $L$. theroni was used to establish adequate intervals for anthelmintic drug administration.
\end{abstract}

Keywords: anthelmintic, chronology, haptor growth, lamellodisc, sclerites

Monogenean monopisthocotyleans from the family Diplectanidae Monticelli, 1903 are characterised by a complex haptor bearing many different attachment elements: two pairs of main hooks joined by medial bars, 14 peripheral marginal hooks and one or two groups of sclerotised rodlets or lamellae called 'squamodiscs' or 'lamellodiscs' (Bychowsky 1957, Desdevises 2001, Domingues and Boeger 2008, Sánchez-García et al. 2011). Within this family, the species of Lamellodiscus Johnston et Tiegs, 1922, and the Lamellodiscinae Oliver, 1969 in general, have one or two lamellodiscs, formed by several overlapping sclerotised plates (lamellae).

Sánchez-García et al. (2011) studied the functional morphology of the haptor of adult Lamellodiscus spp. indicating the roles of each component of this structure in parasite attachment, as well as the pathological implications. According to these authors, the sclerotised lamellae of the ventral and dorsal lamellodiscs slide displaying a telescopic movement, pushing and separating the secondary gill lamellae. This thrust force is opposite to the main hooks' traction force which tightens the epithelia, providing highly efficient attachment and stability for the worms.
The effects on fish of this type of attachment were studied for Lamellodiscus theroni Amine, Euzet et KechemirIssad, 2007 and $L$. falcus Amine, Euzet et Kechemir-Issad, 2006 in the sharpsnout seabream Diplodus puntazzo (Walbaum). In this case, both piercing by hooks and pressure by lamellodiscs were related to some local damage to gills although, in general, the observed effects did not seem severe (Sánchez-García et al. 2011). In contrast, other authors such as Katharios et al. (2006) have described that high burdens of Lamellodiscus spp. can seriously damage cultured sharpsnout seabreams.

This unexpected stronger effect of Lamellodiscus spp. in cultures could be explained by the high parasite numbers and the presence of many different developmental phases occurring together. Each phase often infects different parts of the gill using different attachment mechanisms, subsequently provoking a combined effect of different types of damage to the gills (Repullés-Albelda et al. 2011). Some studies have described details on the development of the haptor of Lamellodiscus spp., providing information about the sequence of development of sclerotised pieces (Bychowsky 1957, Euzet 1957, Oliver 1987). However, the 
attachment strategies have only been described for adults and not for post-larval stages. Knowledge of life cycle chronology is also lacking and it is important to understand attachment and damage dynamics.

The main aim of this work was to study the developmental changes of $L$. theroni over time. To do so, we monitored parasite development under experimental conditions, focusing on the morphological changes in the sclerotised structures. We also studied the changes in monogenean attachment strategies during haptor development, as well as the pathological implications.

\section{MATERIALS AND METHODS}

Forty sharpsnout seabreams infected with Lamellodiscus theroni (further referred to as donor fish) were collected in May 2008 from Mar Menor (37041'N; 0 44'E; Spanish Mediterranean) and confined in the facilities of the Instituto Murciano de Investigación y Desarrollo Agrario y Alimentario (IMIDA) at San Pedro de Pinatar, Murcia, Spain. Parasite infection parameters were assessed by examination of the gills of 24 fresh fish from the facilities of IMIDA using Leica MZ AP0 (8-80×) stereomicroscope. Fish were transported alive and maintained in the aquaria of the Central Service for the Support to Experimental Research of the University of Valencia (SCSIE). Once in the SCSIE facilities, ten fish (mean \pm SD: total length $296 \pm 13 \mathrm{~cm}$; weight $393 \pm 41 \mathrm{~g}$ ) were killed by cervical decapitation to monitor parasite levels (overall prevalence: $100 \%$; mean intensity: 985 parasites/infected fish). Gills were checked for parasites under light microscope. The thirty remaining fish were used as donors. Naive fish were acquired from a Greek hatchery, transported to the SCSIE facilities and reared for three years. All fish were maintained in parasite-free aquaria with marine water at salinity of $37 \%$, temperature of $20^{\circ} \mathrm{C}$, and a photoperiod of $8: 16 \mathrm{~h} \mathrm{light/dark}$.

One hundred and fifty naive fish (total length $171 \pm 13 \mathrm{~mm}$; weight $68.5 \pm 12.5 \mathrm{~g}$ ) were infected by cohabitation with the donor sharpsnout seabreams for $48 \mathrm{~h}$ in a 250 litre tank. After the infection period, fish were transferred to experimental tanks (volume 250 litres). 'Time 0 ' of the experiment was assumed as the moment when fish were restocked in the experimental tanks. The experiment lasted 22 days. From 'time 0 ', five fish were killed daily by cervical decapitation and analysed for presence of monogeneans. From each fish, gills were isolated in individual Petri dishes in seawater and analysed immediately after dissection with Leica MZ AP0 (at magnifications 8-80×) stereomicroscope with transmitted light illumination. Each first lamella was observed carefully, without removing parasites from their infection sites. After examination, gills with higher parasite abundance were fixed in $4 \%$ formalin for histological analysis. Fish skin and fins were also examined for the presence of post-larvae or adults.

Monogeneans were collected, killed in hot saline solution and mounted as temporary slides with glycerin jelly. The sclerotised parts of the haptor and the male copulatory organ were studied with differential interference contrast (DIC) microscopy (Leica DMR, magnifications 100-1000×). Morphological study of the development of $L$. theroni started from the earliest post-larvae, as described by Bychowsky (1957), corresponding to phase I of the present study. The sequence in which each sclerotised element of the haptor appeared was recorded and both shape and growthrelated changes were monitored. The sclerites of $L$. theroni were
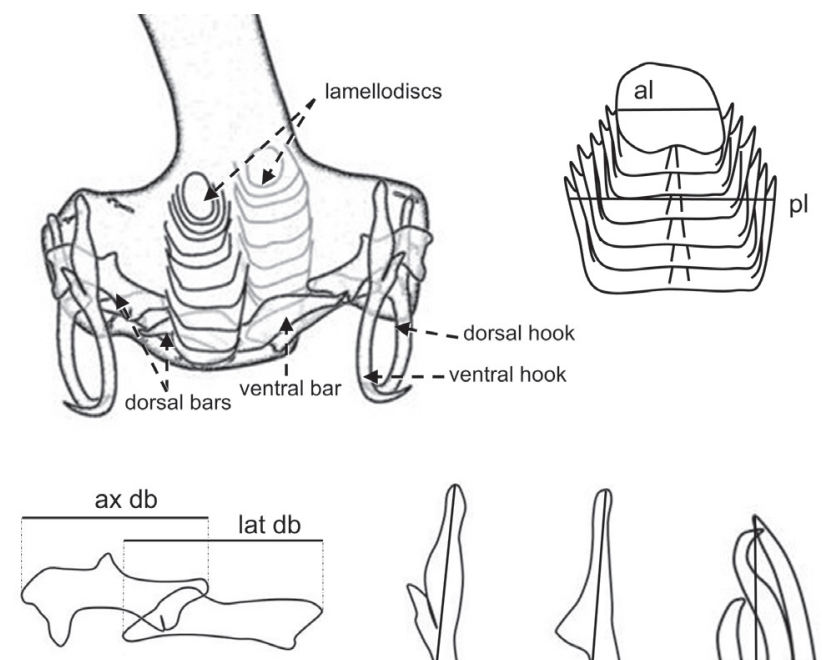

$\mathrm{vb}$
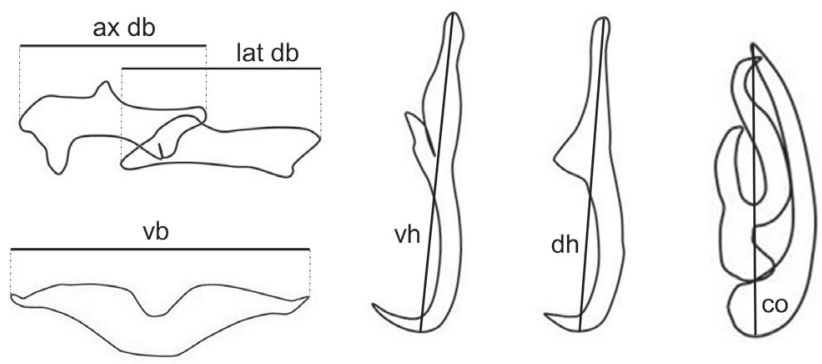

Fig. 1. Haptor of Lamellodiscus theroni Amine, Euzet et Kechemir-Issad, 2007. Measurements taken from the sclerotised pieces. Abbreviations: al - length of the anteriormost lamella; ax $\mathrm{db}$ - length of the axial part of the dorsal bar; co - length of the male copulatory organ; dh - length of the dorsal hook; lat $\mathrm{db}$ - length of the lateral part of the dorsal bar; $\mathrm{pl}$ - length of the posteriormost lamella; vb - length of the ventral bar; vh - length of the ventral hook.

drawn with the help of a drawing tube and measured using Image Tool for Windows v 3.1 (see graphical definition of the measurements in Fig. 1). All measurements are in micrometres unless otherwise indicated. The terminology for the different parts of haptor sclerites follows that of the original description of the species (Amine et al. 2007).

For the histological study, infected gill arches previously fixed in $4 \%$ formalin were embedded in paraffin, serially sectioned at $4 \mu \mathrm{m}$ with a rotary microtome, stained with haematoxylin and eosin, mounted in Entellan ${ }^{\circledR}$ (Merck, Darmstadt, Germany) and observed under light microscopy (Leica DMR HC microscope).

\section{RESULTS}

\section{Developmental stages}

All parasites were found on gills, none on skin or fins. In total 64 parasites were collected, including post-larvae and adults (prevalence $56 \%$ and mean intensity 1.6). Oncomiracidia needed at least 15 d.p.i. to develop into adults (phase VI) and 18 days post infection (d.p.i.) to mature and develop eggs. The second generation of post-larval specimens was found at 22 d.p.i. Table 1 shows the measurements of the body and sclerotised elements of the haptor during the development and Fig. 2 provides graphical illustration of the morphological changes in haptoral sclerites and sites of attachment of the specimens of $L$. theroni studied. Six developmental phases were distinguished in the development of $L$. theroni, according to the sequential formation of the elements of the haptor (Fig. 2). These are described below. 
Table 1. Lengths of the body and sclerotised structures during the development of Lamellodiscus theroni Amine, Euzet et KechemirIssad, 2007 from Diplodus puntazzo (in micrometres).

\begin{tabular}{lcccccc}
\hline Structure / Phase & I & II & III & IV & V & VI \\
& $\mathrm{n}=8$ & $\mathrm{n}=8$ & $\mathrm{n}=2$ & $\mathrm{n}=20$ & $\mathrm{n}=21$ & $\mathrm{n}=14$ \\
\hline Body & $144-182$ & $152-170$ & $1585-159$ & $163-281$ & $258-509$ & $475-888$ \\
Peripheral marginal hooks & $7-9$ & $7.5-10$ & $8.5-10.5$ & $8-10$ & $8.5-11.5$ & $8-10.5$ \\
Ventral hook & - & $39-66$ & $63-84$ & $81-99$ & $112-136$ & $115-141$ \\
Dorsal hook & - & $28-45$ & $35-59$ & $55-89$ & $103-127$ & $111-125$ \\
Ventral bar & - & - & $44-55$ & $61-79$ & $103-163$ & $143-178$ \\
Dorsal lateral bar (axial/lateral) & - & - & - & $39-57$ & $70-92 / 58-81$ & $82-93 / 71-89$ \\
Lamellodisc width (internal/external) & - & - & - & - & $22-30 / 49-70$ & $22-30 / 53-66$ \\
Male copulatory organ & - & - & - & - & - & $36-94$ \\
\hline
\end{tabular}

Phase I (1-12 d.p.i.; $\mathrm{n}=15$ ) (Figs. 2, 3): tiny first postlarval stages just after fixation on gills, very similar to oncomiracia but without cilia. Post-larvae characterised by haptor armed only with marginal hooks (also known as uncinuli). Body elongated and slightly dorsoventrally flattened. Haptor with 14 sclerotised short hooks (further referred to as peripheral marginal hooks); 12 of these disposed circularly along haptor edge and one pair located at haptor centre. Each hook with thick handle and distal pointed and hooked tip. Hook shape does not change along parasite life, but hooks gradually become slightly longer in time. Two pairs of eyespots situated on anterior part of body, two anterior smaller and subspherical to pyriform and two posterior larger and kidney-shaped. Eyespots present during whole parasite life.

Phase II (5-15 d.p.i.; $\mathrm{n}=14$; more than $50 \%$ of postlarvae reach to this phase at day 8) (Figs. 2, 4A,B): postlarvae characterised by appearance of main hooks (ventral and dorsal; also known as anchors). Total body length very similar to that of previous phase. Haptoral region notably distinct, wider and longer (approximately half body length). Peripheral marginal hooks as in previous phase, except for two central, gradually relocated further apart from centre to haptor, at posterior end (Figs. 3A, 4A). Dorsal and ventral pairs of hooks larger than peripheral marginal from first appearance, formed by thin straight bars with hooked posterior end. Ventral hooks approximately $30 \%$ longer than dorsal hooks. Shape of dorsal and ventral hooks almost invariable during this phase, length slightly increasing.

Phase III (7-8 d.p.i.; $\mathrm{n}=2$ ) (Figs. 2, 4A): post-larvae characterised by appearance of medial ventral bar. Total body length still very similar to previous phases. Main hooks distinctly longer and more robust, ventral hooks approximately one third longer than dorsal. Ventral hooks at early stage of their final appearance, developing anterior protuberance that will become hook 'guard' (Fig. 2). Early ventral bar small and thin single piece, slightly bent and pointed at both ends, arranged perpendicularly to main hook; length still half that of adult.

Phase IV (8-16 d.p.i.; $\mathrm{n}=21$, more than $50 \%$ of postlarvae reach to this phase at day 11) (Figs. 2, 4A): postlarvae characterised by the appearance of the lateral dorsal bars. First noticeable body length increase occurred at this phase; dorsal and ventral hooks enlarge, appearing more robust. Early 'guards' perceptible in both pairs of hooks, differing slightly between ventral and dorsal hooks: guard projections of ventral hooks elongated and anteroventroally oriented, guard projections of dorsal hooks appearing as short ventral swellings, becoming more apparent in later stages. Ventral bar thin and curved, longer but similar in shape to that of previous stage. Early dorsal bars only composed of a pair of single small elongated sclerites (left and right), perpendicular to oblique to ventral bar, which appear to be axial parts. Dorsal bars noticeably growing during this phase, even developing distal protuberance, slightly similar to that in axial part in adults.

Phase $\mathbf{V}$ (9-16 d.p.i.; $\mathrm{n}=20$, more than $50 \%$ of postlarvae reach to this phase at day 14) (Figs. 2, 4A,C): postlarvae characterised by appearance of dorsal and ventral lamellodiscs. Worm total length increases to approximately double previous phase size. All haptoral elements present. Peripheral marginal hooks located at haptor edge. Dorsal and ventral hooks similar, with almost definitive adult length and thickness, very similar to those of adults. Posteroventral hook guards well defined, allowing differentiation between dorsal and ventral pairs; guard as oblique digitiform projection in ventral hooks and soft swelling in dorsal. Ventral bar with almost definitive length and shape; lateral ends becoming gradually more robust, leaving medial bend as narrow notch, typical of adult ventral bar. Dorsal bars developing into two overlapping pieces, axial and lateral parts. Dorsal bars still thin, developing the species-distinctive protuberances, while their arrangement gradually turns from oblique to almost perpendicular to longitudinal axis (lined up with ventral bar). Early lamellodiscs located at dorsal and ventral sides, anterior to other sclerites. Not all lamellae distinguishable at this stage (4-8 lamellae visible in each lamellodisc), appearing as soft narrow primordial plates, except for already circular anteriormost lamella.

Phase VI (from 15 d.p.i.; $\mathrm{n}=23$, more than $50 \%$ of monogeneans reach this phase at day 15) (Figs. 2, 5A): adult worms characterised by appearance of male copulatory organ. Haptor completely developed, with all sclerotised elements reaching their definitive size and shape. Body elongation and haptor lateral growth, giving final T-shape to body. Dorsal and ventral pairs of hooks and dorsal and ventral bars acquiring their final arrangement, shape and thickness. Lamellodisc area representing $1 / 12$ of 


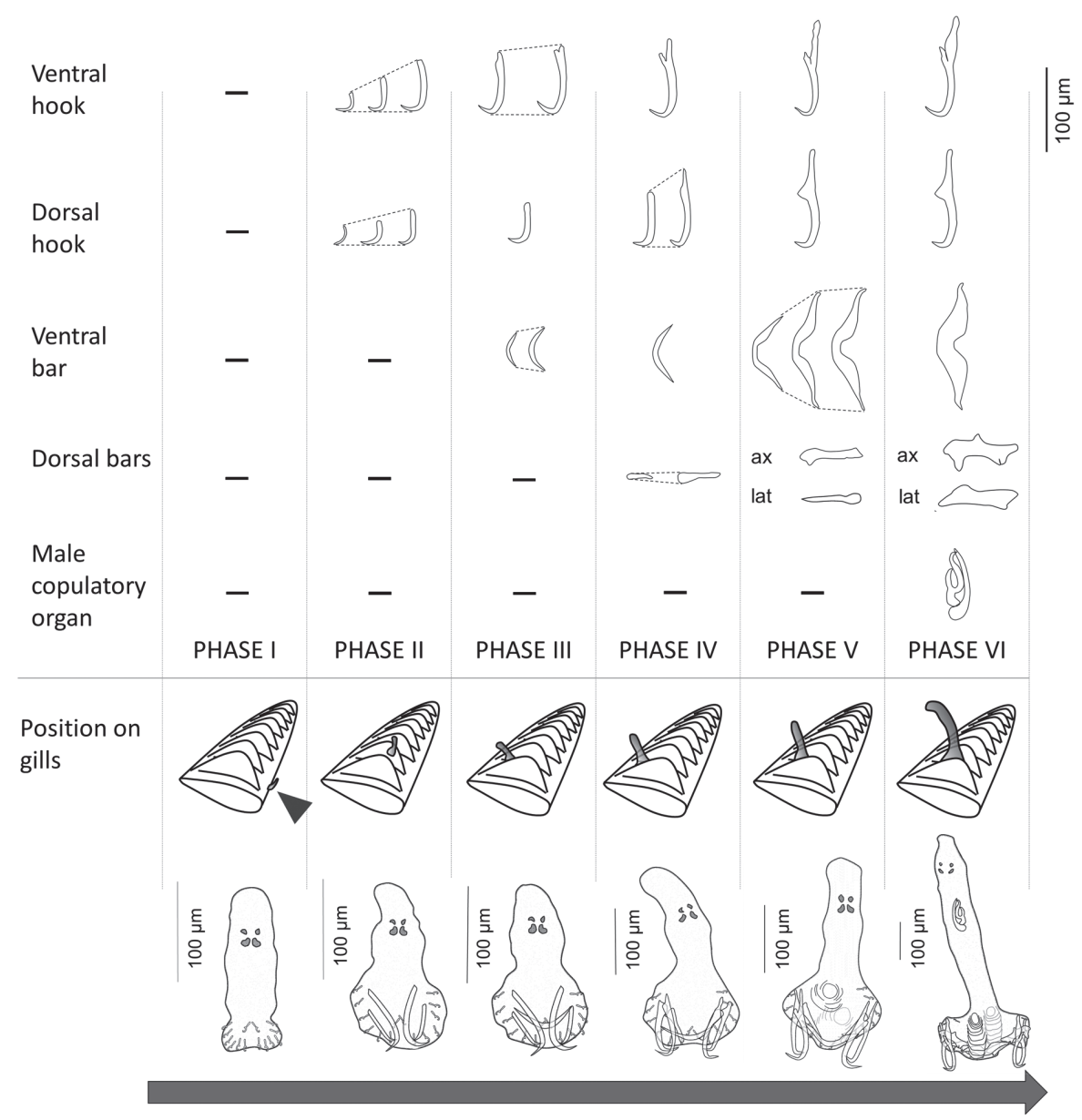

Fig. 2. Phases of development of Lamellodiscus theroni Amine, Euzet et Kechemir-Issad, 2007. Morphological changes of the sclerotised structures and attachment places of Lamellodiscus theroni parasitising Diplodus puntazzo (Walbaum) during development. The black arrow indicates the position on the gill lamella of $L$. theroni corresponding to phase I.

total body length. Lamellae fully developed. Male copulatory organ composed of two species-specific sclerotised, articulated pieces, with impair piece with curved tip. First intrauterine eggs detectable at 18 d.p.i.

\section{Attachment development}

The analysis of the histological sections revealed that parasites modified their attachment mechanism depending on the available functional sclerotised structures present in the haptor. During phase I, the only haptoral sclerites were the peripheral marginal hooks; at this phase the haptor is a flattened surface with 14 small marginal hooks more or less uniformly arranged around the haptor (except for the central ventral pair) and mostly oriented to the ventral side (Fig. 3). Histological sections showed that parasites used the marginal hooks to attach mainly on the flattened surfaces of the gill filaments or between the secondary gill lamellae. When L. theroni were attached on flattened surfaces, all marginal hooks showed the same ventral orientation (Fig. 3E). Nevertheless, we observed in some slides that some marginal hooks were orient dorsally (Fig. 3D) attaching the monogenean onto two contiguous lamellae.

During phases II, III and IV, the components of the haptor gradually appeared and took part in the attachment. These stages were usually found between the secondary gill lamellae, normally quite external and far from the basal lamina (Fig. 4). At phase II the sizes of dorsal hooks appeared to be too small and tips did not manage to pierce the gill tissue (Fig. 4B). Meanwhile, ventral hook tips protruded from the haptor tegument, reaching and piercing the tissue of the same secondary gill lamella. Dorsal hooks started to take part in the attachment at phase III; at this moment the ventral hooks were attached to secondary gill lamellae while the dorsal hooks, oriented backwards, pierced the contiguous secondary gill lamella, pulling the gill lamellae towards the parasites. This type of attachment is facilitated at phase IV by the longer and more robust main hooks, which penetrate deeper into gill tissues. During phases II, III and IV, the peripheral marginal hooks were seen to be orientated dorsally and ventrally, hooking two contiguous lamellae of interlamellar spaces (Fig. 4B).

At phase $\mathrm{V}$, the lamellae of the lamellodiscs were thin and narrow, apparently non-functional (Fig. 4C). They became gradually thicker and larger, acquiring the definitive capability to slide performing a telescopic movement at phase VI (Fig. 5). During this growth period, the lamellodiscs could gradually push the secondary lamellae further. Finally, the adult worms became attached via the previously described mechanism of pushing the tissues with dorsal and ventral lamellodises while pulling with the dorsal and 


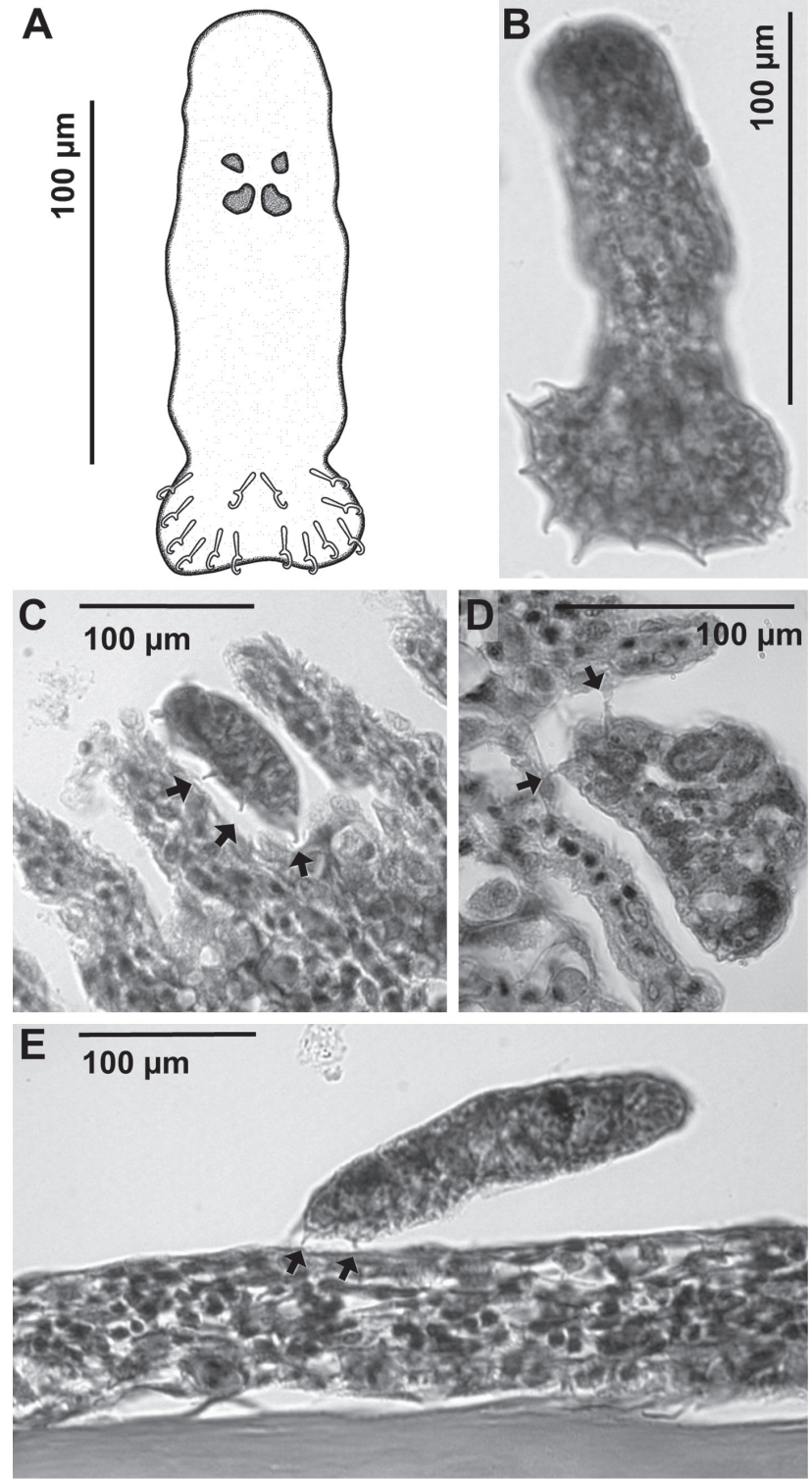

Fig. 3. Development of Lamellodiscus theroni Amine, Euzet et Kechemir-Issad, 2007 on the gills of Diplodus puntazzo (Walbaum), Phase I. A - drawing of the whole worm; B-E - histological sections obtained from parasitised gills. C, D-parasites were found within the interlamelar space; $\mathbf{E}$ - parasites were found on a flat surface of a first lamella. Black arrows point to the peripheral marginal hooks.

ventral hooks. At phase VI, peripheral marginal hooks are very small in relation to body length, but were observed to contribute to attachment piercing ventral and dorsal tissues.

\section{DISCUSSION}

Most of the studies on monogenean development (including that of Lamellodiscus elegans Bychowsky, 1957 described by Bychowsky 1957) refer to morphological changes in relation to body growth, rather than considering the timing of these changes (Prost 1963, Kearn 1968, Ogawa 1988, Roubal and Diggles 1993, Dzika et al. 2009). Growth of monogeneans seems to follow different developmental timing, depending on the biological needs at each stage (Repullés-Albelda et al. 2011). The present study indicates that in Lamellodiscus theroni total and partial growth varies in time, in order to satisfy its biological demands along the development.

The development of $L$. theroni is, in general, very similar to that of other species of diplectanids as well as to species of other closely related families such as tetraonchids and dactylogyrids (Bychowsky 1957, Euzet 1957, Euzet and Audouin 1959, Kearn 1968, Oliver 1987). However, some specific differences were observed in this species. Compared with previous studies on the development of $L$. elegans, the earliest post-larvae, corresponding to phase I in L. theroni, were longer (144-182 $\mu \mathrm{m}$ vs 60-70 $\mu \mathrm{m}$ long; see Bychowsky 1957). The development of the sclerotised pieces of the haptor followed the same order previously described by Bychowsky (1957), although differences were observed in the rates of development of some structures. The size of the main pairs of hooks (dorsal and ventral) of L. theroni continuously increased from phase II to VI.

Interestingly, Bychowsky (1957) reported that the main hooks were characterised by rapid growth, acquiring their definitive size and shape in phase IV. This difference could be related to the variable dimensions of the main hooks in adults of both species, which are proportionally larger in L. theroni than in L. elegans. As previously described by Bychowsky (1957), all lamellae of the lamellodiscs of L. elegans were simultaneously noticeable, although lamellae were incomplete and thin in the phase $\mathrm{V}$ of $L$. theroni. The body growth of $L$. theroni was relatively slow during the first five phases, body length increasing to $130 \%$ of initial size (see Table 1), while from phase V to VI body length of the specimens increased to $205 \%$ of initial size.

Therefore, the fastest growth occurred when all the sclerites were present in the haptor. This is similar to the growth pattern described in other monogenean, Sparicotyle chrysophrii (van Beneden et Hesse, 1863) (see Repullés-Albelda et al. 2011). The early life of monogeneans is a critical period when main parasite investments are spent on the development of the haptor components, thus guaranteeing a lasting and stable attachment to the fish surface tissues. Once the attachment structures are effectively operating, they can secure attachment of larger parasites to the host tissues and parasites can grow and reproduce. Similarly, Kearn (1968) reported that the diplectanid Diplectanum aequans Wagener, 1857 experiences morphological changes during the development to change from being attached to the skin at the earliest stages of development to the attachment in their definitive habitat, the interlamellar space. Interestingly, L. theroni was always found on gills, not on the skin, which is in contrast with Bychowsky's (1957) report for L. elegans. According to Repullés-Albelda et al. (2011), when parasites invest more resources in reproduction, growth slows down again. This tendency was not explored herein as the experiment was stopped when parasites reached mature stage.

The histological study of $L$. theroni showed that the short ventral marginal hooks lie over the gill tissues, piercing them superficially. This simple attachment mechanism seems to be sufficient to attach these small parasites. Moreover, their small body size allows them to fit in any gill 

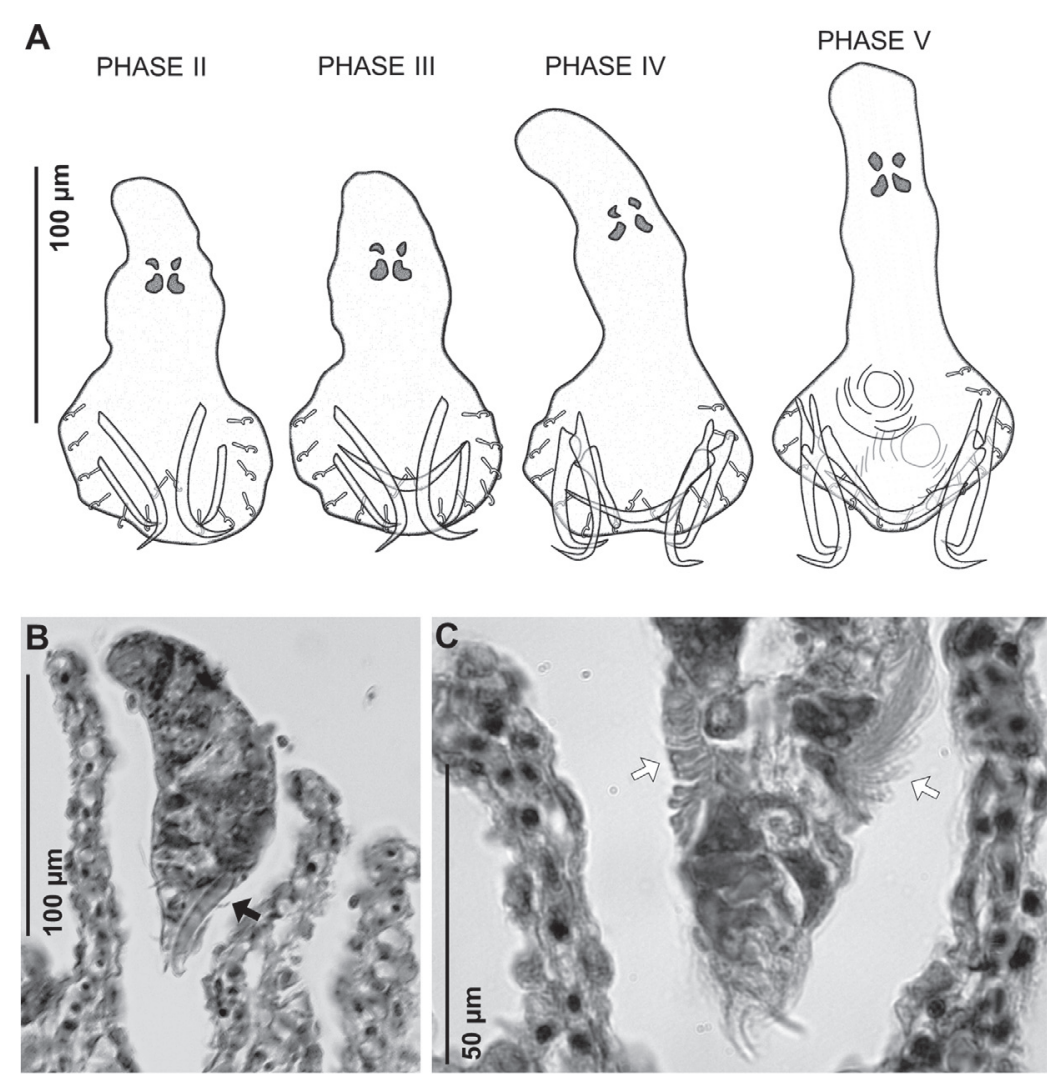

Fig. 4. Development of Lamellodiscus theroni Amine, Euzet et Kechemir-Issad, 2007 on the gills of Diplodus puntazzo (Walbaum), Phases II-V. A - drawings of whole worms; B, C - histological sections of specimens attached between two secondary lamellae. The black arrow indicates the ventral hook of a Phase II individual and white arrows indicate the rudimentary lamellodiscs of Phase V individuals.
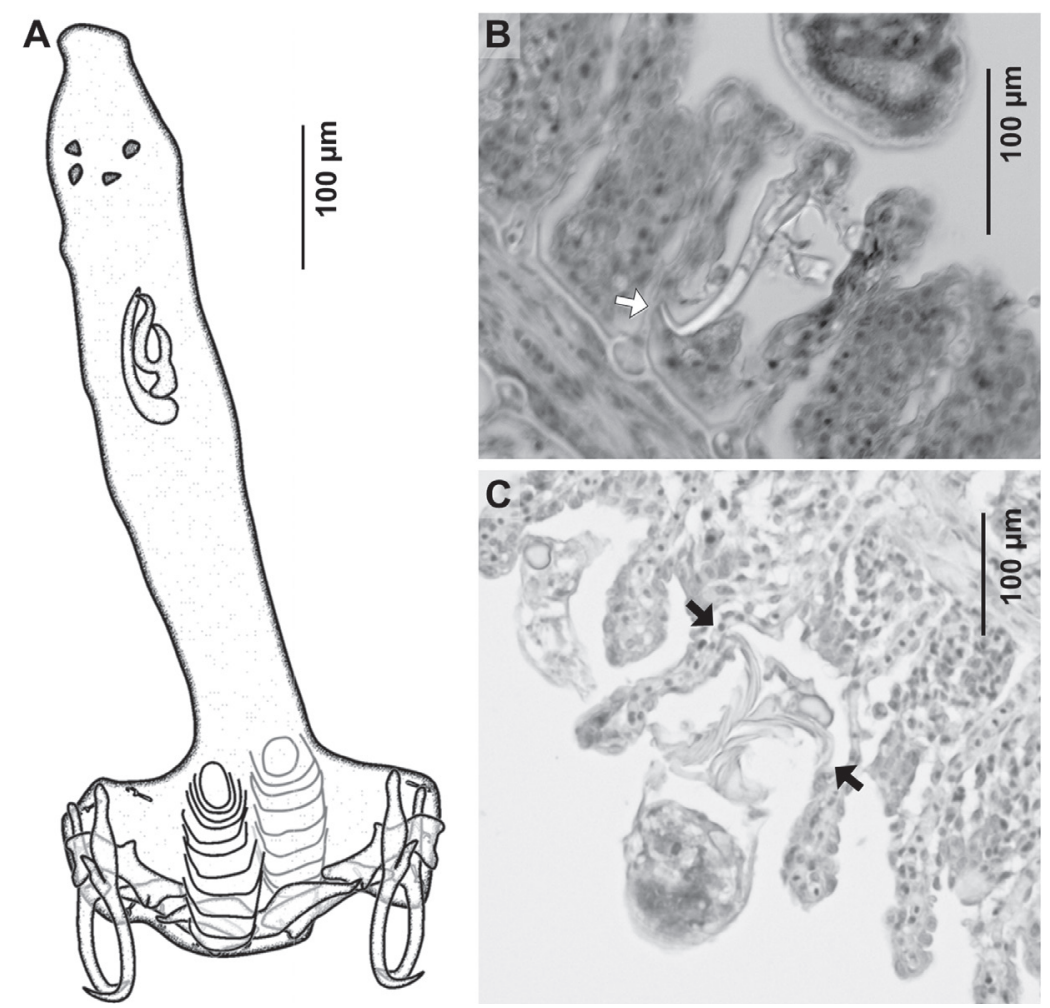

Fig. 5. Development of Lamellodiscus theroni Amine, Euzet et Kechemir-Issad, 2007 on the gills of Diplodus puntazzo (Walbaum), Phase VI. A - drawing of whole worm; B, C - histological sections of specimens attached deeply inside the interlamellar space. Black arrow indicates the dorsal hook piercing the gill epithelium and white arrows indicates to the gill epithelium pushed by the dorsal and ventral posteriormost lamellae of the lamellodiscs. 
habitat, usually being found on flat smooth surfaces. Some parasites of phase I also penetrated deeply into the interlamellar space. In this case, some marginal hooks could be oriented posteriorly or dorsally to pierce the surrounding gill surfaces (Fig. 3). This observation agrees with that of Sánchez-García et al. (2011), who described the movements of the marginal hooks of Lamellodiscus spp. as multidirectionally pendular. The non-selective attachment together with the small size of the parasites increase the diversity of the gill areas that the earliest post-larvae of $L$. theroni can colonise, favouring their displacement along the gill filaments. Other strategies, such as the secretion of adhesive substances, can also be involved in parasite attachment at this and the following phases (Paling 1966).

As parasite body size increases, new more stable attachment mechanisms develop, being proportional in size to total parasite length. The appearance of the main hooks means an important change in parasite attachment strategy; these long hooks pierce fish tissues deeply while the peripheral marginal hooks become accessory attachment structures. Although ventral and dorsal hooks were detected simultaneously, only ventral hooks seemed to be functional at phase II, as dorsal hooks were short and did not exceed haptor tegument. Histological sections showed that parasites lay on their ventral sides. From phase III on, both dorsal and ventral hooks were functional, piercing the contiguous secondary lamellae besides the interlamellar space, as described for adults by Sánchez-García et al. (2011). Ventral and dorsal bars did not initially take part in the attachment. When the adult haptor of $L$. theroni acquires the transversely-elongated T-shape, these bars are thick and robust, dorsal bars are aligned with the ventral bar and perpendicular to the longitudinal body axis, probably improving the skeletal support and providing fastening points for the hook musculature (see e.g. musculature arrangement in Diplectanum aequans in Paling 1966).

According to Sánchez-García et al. (2011) the attachment of the adult Lamellodiscus spp. also requires the thrust force of the evertible lamellodiscs, opposite to the traction force of the main hooks. However, the histological sections of $L$. theroni showed that the 'early' lamellodiscs (phase V) were incomplete and thin, i.e. not large enough to push gill lamellae apart (Fig. 4C). They enlarge during phase VI until becoming functional, contributing to more efficient attachment of the larger worms.

The monopisthocotylean attachment system based on hooks has been considered particularly pathogenic (Whittington and Chisholm 2008). However, the degree of damage depends on the attachment strategies during the distinct post-larval stages of each species. Effects of Lamellodiscus spp. on fish are usually considered as mild, although Katharios et al. (2006) reported mortalities of sharpsnout seabreams due to infection with parasites of this genus. In our experiment, the first post-larval monogeneans were attached by the tiny marginal hooks, piercing quite superficially and not related to noticeable lesions. The main hooks are larger thus increasing the number and severity of intrusive lesions; dorsal and ventral hooks can penetrate deep inside the epithelia and sometimes perforate the blood vessels, causing small hemorrhages (Sánchez-García et al. 2011). Gill damage becomes even worse when lamellodiscs begin to take part in the attachment, tightening and increasing their contact with gill tissues.

Species of Lamellodiscus are common gill parasites of Mediterranean cultured sparids. The confinement of farmed fish facilitates reinfections, leading to high parasite loads (Katharios et al. 2006, Mladineo 2006, Toksen 2006, Dezfuli et al. 2007, Sánchez-García et al. 2013), entailing a high number of intrusive lesions and consequently invoking more serious respiratory problems (Katharios et al. 2006, Sánchez-García et al. 2011). Moreover, we must consider that fish simultaneously harbour different developmental stages, each having different attachment strategies and microhabitat preferences; therefore, gill damage is more widely extended.

Knowledge of key developmental events can also help establish adequate intervals during which anthelmintic treatment is administ, as parasites inside eggs can survive such treatments (Repullés-Albelda et al. 2012). In this study, L. theroni became gravid from 18 d.p.i. on at $20^{\circ} \mathrm{C}$, and a second generation was found on gills at 22 d.p.i. A two-dose treatment, separated by a minimum of four days, could be optimal for long-lasting deworming. In this way, post-larvae and adults would be eliminated by the first dose, while the second dose should be administered after all new worms have hatched from the surviving eggs. Effects of abiotic factors must be studied because developmental periods could be altered by environmental conditions (Mo 1993, Rubio-Godoy and Tinsley 2002, Kearn 2004), mainly temperature (Cecchini 1994, Cecchini et al. 1998).

In conclusion, the deep changes of the haptor during parasite development lead to sequential changes of attachment mechanisms and related damage. The present study can also be useful as a starting point to design effective anthelmintic applications. Other aspects such as the effect of abiotic factors, parasite intensity or the presence of synergistic gill parasites must be strongly considered in further studies. The study of the development of other species of Lamellodiscus may provide comparative information in order to identify which aspects can affect timing and infection patterns.

Acknowledgements. We are indebted to the Support to Experimental Research of the University of Valencia (SCSIE). We are grateful to Benjamín García and Jesús Cerezo from the Instituto Murciano de Investigación y Desarrollo Agrario y Alimentario of the Region of Murcia. We are also grateful to the editors for their invaluable suggestions. N.S.-G. benefits from a 'V Segles' $\mathrm{PhD}$ student grant awarded by the University of Valencia. This study was supported by projects PROMETEO 2011-040 and ISIC/2012/003 of the Valencian Local Government; AGL200501221 and AGL 2012-20892 of the Spanish Ministry of Science and Innovation. Thanks are due to F. Barraclough for revising the English. 


\section{REFERENCES}

Amine F., Euzet L., Kechemir-Issad N. 2007: Lamellodiscus theroni sp. nov. (Monogenea, Diplectanidae), a gill parasite from Diplodus puntazzo (Teleostei, Sparidae) from the Mediterranean Sea. Acta Parasitol. 52: 305-309.

Bychowsky B.E. 1957: Monogenetic Trematodes. Their Systematics and Phylogeny. English translation. American Institute of Biological Sciences, Washington, D.C., 1961, 627 pp.

CECCHIN S. 1994: Influence of temperature on the hatching of eggs of Diplectanum aequans, a parasite of sea bass. Aquacult. Int. 2: 249-253.

Cecchini S., Saroglia M., Berni P., Cognetti-Varriale M. 1998: Influence of temperature on the life cycle of Diplectanum aequans (Monogenea, Diplectanidae), parasitic on sea bass, $D i$ centrarchus labrax (L.). J. Fish Dis. 21: 73-75.

Desdevises Y. 2001: The phylogenetic position of Furnestinia echeneis (Monogenea, Diplectanidae) based on molecular data: a case of morphological adaptation? Int. J. Parasitol. 31: 205-208.

Dezfuli B.S., Giari L., Simoni E., Menegatti R., Shinn A.P., Manera M. 2007: Gill histopathology of cultured European sea bass, Dicentrarchus labrax (L.), infected with Diplectanum aequans (Wagener 1857) Diesing 1958 (Diplectanidae: Monogenea). Parasitol. Res. 100: 707-713.

Domingues M.V., Boeger W.A. 2008: Phylogeny and revision of Diplectanidae Monticelli, 1903 (Platyhelminthes: Monogenoidea). Zootaxa 1698: 1-40.

Dzika E., Dzikowiec M., Hoffmann R.W. 2009: Description of the development of the attachment and copulatory apparatus of Dactylogyrus extensus from Cyprinus carpio var. koi. Helminthologia 46: 39-44.

Euzet L. 1957: Recherches sur les Monogenoidea parasites de poissons marins. Ann. Parasitol. Hum. Comp. 32: 469-481.

Euzet L., Audouin J. 1959: Sur un genre nouveau de Monogenoidea parasite de la dorade Chrysophrys aurata. L. Rev. Trav. Inst. Pêches Marit. 23: 317-322.

Katharios P., Hayward C., Papandroulakis N., Divanach P. 2006: Pathology of Lamellodiscus spp. (Monogenea) parasitizing the gills of sharpsnout seabream and preliminary results of formalin treatment. Bull. Eur. Assoc. Fish Pathol. 26: 196-201.

KeARN G.C. 1968: The development of the adhesive organs of some diplectanid, tetraonchid and dactylogyrid gill parasites (Monogenea). Parasitology 58: 149-163.

KeARn G.C. 2004: Monogenean gill parasites, polyopisthocotyleans. In: G.C. Kearn (Ed.), Leeches, Lice and Lampreys. A Natural History of Skin and Gill Parasites of Fishes. Springer, Norwell, pp. 103-130.

Mladineo I. 2006: Parasites of Adriatic cage reared fish. Acta Adriat. 47: 23-28

Mo T.A. 1993: Seasonal variations of the opisthaptoral hard parts of Gyrodactylus derjavini Mikailov, 1975 (Monogenea: Gyrodactylidae) on brown trout Salmo trutta L. parr and Atlantic salmon
S. salar L. parr in the River Sandvikselva, Norway. Syst. Parasitol. 26: 225-231.

Ogawa K. 1988: Development of Bivagina tai (Monogenea, Microcotylidae). Nippon Suisan Gakkaishi 54: 61-64.

Oliver G. 1987: Les Diplectanidae Bychowsky 1957 (Monogenea, Monopisthocotylea, Dactylogyridea). Systématique, biologie, ontogénie, écologie, essai de phylogénèse. Thèse d'état, Université des Sciences et Techniques du Languedoc, Montpellier, 433 pp.

PaLing J.E. 1966. The attachment of the monogenean Diplectanum aequans (Wagener) Diesing to the gills of Morone labrax L. Parasitology 56: 493-503.

Prost M. 1963: Investigations on the development and pathogenicity of Dactylogyrus anchoratus (Duj. 1845) and D. extensus Mueller et v. Cleave, 1932 for breeding carps. Acta Parasitol. Pol. 11: $17-47$.

Repullés-Albelda A., Holzer A.S., Raga J.A., Montero F.E. 2012: Oncomiracidial development, survival and swimming behaviour of the monogenean Sparicotyle chrysophrii (van Beneden and Hesse, 1863). Aquaculture 338-341: 47-55.

Repullés-Albelda A., Raga J.A., Montero F.E. 2011: Postlarval development of the microcotylid monogenean Sparicotyle chrysophrii (van Beneden and Hesse, 1863): comparison with species of Microcotylidae and Heteraxinidae. Parasitol. Int. 60: 512-520.

Roubal F.R., Diggles B.K. 1993: The rate of development of Polylabroides multispinosus (Monogenea: Microcotylidae) parasitic on the gills of Acanthopagrus australis (Pisces: Sparidae). Int. J. Parasitol. 23: 871-875.

Rubio-Godoy M., Tinsley R.C. 2002: Trickle and single infection with Discocotyle sagittata (Monogenea: Polyopisthocotylea): effect of exposure mode on parasite abundance and development. Folia Parasitol. 49: 269-278.

Sánchez-García N., Ahuir-Baraja A.E., Raga J.A., MonteRO F.E. 2013: Morphometric, molecular and ecological analyses of the parasites of the sharpsnout seabream Diplodus puntazzo Cetti (Sparidae) from the Spanish Mediterranean: implications for aquaculture. J. Helmintol. 3: 1-15.

Sánchez-García N., Padrós F., Raga J.A., Montero F.E. 2011: Comparative study of the three attachment mechanisms of diplectanid monogeneans. Aquaculture 318: 290-299.

TOKSEN E. 2006: Occurrence of gill parasite Lamellodiscus ignoratus (Palombi, 1943) (Monogenea: Diplectanidae) in cultured sharp-snout sea bream, Diplodus puntazzo (Cetti, 1777) from Izmir, Turkey. Bull. Eur. Assoc. Fish Pathol. 26: 174-179.

Whittington I.D., Chisholm L.A. 2008: Diseases caused by Monogenea. In: J.C. Eiras, H.S.T. Wahlii and B.G. Kapoor (Eds.), Fish Diseases. Science Publishers, New Hampshire, pp. 683-816. 